\title{
Deficiencies in education and experience in the management of acute kidney injury among Malawian healthcare workers
}

\section{R Evans ${ }^{1,2}$, P Rudd $^{2}$, U Hemmila ${ }^{2}$, H Dobbie $^{3}$, G Dreyer ${ }^{2,3}$}

1. Department of Medicine, College of Medicine, University of Malawi, Blantyre, Malawi

2. Department of Medicine, Queen Elizabeth Central Hospital, Blantyre, Malawi

3. Nephrology Department, Bart's Health, London, United Kingdom

Correspondence to: Rhys DR Evans,

E-mail: rhysdrevans@gmail.com

Abstract

\section{Background}

Acute kidney injury (AKI) is a common but under-recognised disease process, which carries a high risk of mortality or chronic complications, such as chronic kidney disease and other organ dysfunction. Management of AKI, however, is suboptimal, both in developed settings and in Malawi. This is partly because of deficiencies in AKI education and training.

\section{Aim}

To establish current levels of AKI education in a range of healthcare workers in Malawi.

\section{Methods}

An AKI symposium was held in Blantyre in March 2015. Delegates were asked to complete a survey at the start of the symposium to assess their clinical experience and education in the management of AKI.

\section{Results}

From 100 delegates, 89 nurses, clinical officers, and physicians, originating from 11 different districts, responded to the survey. Twenty-two percent of healthcare workers (including $28 \%$ of district workers of the various cadres and $31 \%$ of nurses) had never received teaching on any aspect of renal disease, and $50 \%$ (including $63 \%$ of district workers and $61 \%$ of nurses) had never received teaching specifically on AKI. Forty-four percent did not feel confident managing AKI, and 98\% wanted more support managing patients with renal disease. Thirty-four percent (including $55 \%$ of district workers) were unaware that haemodialysis was available at Queen Elizabeth Central Hospital (QECH) for the treatment of AKI and $53 \%$ ( $74 \%$ of district workers) were unaware that peritoneal dialysis was available for the treatment of AKI in children. Only 33\% had ever referred a patient with AKI to QECH.

\section{Conclusions}

There are deficiencies in education about, and clinical experience in, the management of AKI among Malawian healthcare workers, in addition to limited awareness of the renal service available at QECH. Urgent action is required to address these issues in order to prevent morbidity and mortality from AKI in Malawi.

\section{Introduction}

Acute kidney injury (AKI) is a rapid worsening or loss of kidney function that can be caused by a variety of different mechanisms. It is a common and under-recognised disease process, which carries a high risk of mortality or chronic complications, such as chronic kidney disease (CKD) and other organ dysfunction. ${ }^{1}$ There are limited data on the incidence of AKI, particularly in sub-Saharan Africa. From the data available in developed world settings, it is estimated that AKI affects $1 \%$ to $4.3 \%$ of patients presenting to hospital, and at least $7 \%$ of admissions during their inpatient stay. ${ }^{2,3} \mathrm{~A}$ recent meta-analysis, including 154 studies of AKI that were based strictly on a Kidney Disease Improving Global Outcomes (KDIGO) definition of AKI, suggested that the incidence of hospital-acquired AKI (h-AKI) may be as high as $20 \%{ }^{4}$
A study is underway at Queen Elizabeth Central Hospital $(\mathrm{QECH})$, in Blantyre, to assess the incidence, aetiology, and outcomes of community-acquired AKI in Malawi, with the results targeted for publication by the end of the year. AKI has both short- and long-term adverse effects for the individual. It is associated with increased length of hospital stay, and has a wider socioeconomic impact on healthcare systems and societies, with the cost per AKI admission in developed settings estimated to be USD $\$ 7500 .{ }^{5}$ Moreover, it carries a significant mortality rate. In a recent large metaanalysis of all AKI studies from 2004 to 2012, the overall all-cause mortality was $23 \%(23.9 \%$ in adults and $13.8 \%$ in children) with an odds ratio (OR) for death of 4.95 when comparing AKI to non-AKI. ${ }^{4}$ In addition, as the severity of AKI increases, so does mortality, with the greatest risk in those requiring renal replacement therapy (RRT) $(50-60 \%$ mortality overall). Indeed, recent estimates conclude that the death rate per year from AKI is greater than prostate cancer, breast cancer, heart failure, and diabetes. ${ }^{1}$ In addition to excess mortality, there is mounting evidence that AKI is a risk factor for the development of $\mathrm{CKD}$, and the associated cardiovascular risk that this entails. The majority of AKI may be prevented or managed by relatively simple measures, such as fluid resuscitation, identification and treatment of the underlying condition (e.g., sepsis and antibiotics), stopping nephrotoxic drugs, and exclusion of obstruction. Being aware of these relatively basic interventions may lead to reversal of abnormal renal function in a significant proportion of cases of AKI. Despite this, AKI is managed poorly. A recent audit at QECH demonstrated that only $11 \%$ of acute medical admissions had a serum creatinine taken and processed postadmission over a four-week period in $2015 .{ }^{6}$ The exact reasons for these deficiencies in management are unclear but may be, in part, because of a lack of education, at all levels within our healthcare system, regarding the detection and management of AKI. The aim of this study was to assess healthcare workers training in, and experience of, managing AKI in a range of healthcare settings across Malawi.

\section{Methods}

In March 2015 we held an AKI symposium in Blantyre with the aim of raising awareness and improving management of AKI in Malawi. We invited nursing staff, clinical officers, and doctors from all district hospitals in southern Malawi, in addition to health centre workers from within Blantyre, and nurses and clinicians from QECH and Kamuzu Central Hospital $(\mathrm{KCH})$ in Lilongwe. This was achieved by directly contacting the district medical and health officers in each district in Malawi's Southern Region to inform them of the symposium. We then asked them to nominate five to eight interested representatives from their districts to attend. We encouraged these representatives to be from both nursing and medical backgrounds. We also contacted heads of departments at QECH (paediatrics, obstetrics, surgery, ICU, and emergency medicine) to nominate representatives in a similar fashion, in addition to the renal unit at $\mathrm{KCH}$. The individual heads of department and the district medical 
and health officers determined who attended from their respective regions. The district medical officer from Nkhata Bay (Northern Region) also attended, as he had a special interest in renal medicine and became aware of the course while collaborating with the course organizers on another project. The course was held over two days with a mixture of didactic lectures and small group sessions, with case discussions based around real-life examples of AKI from QECH. An international faculty of 10 nephrologists delivered the curriculum. At the start of the course, we conducted a survey to assess educational exposure to, and clinical experience of, managing AKI. We invited all delegates present to respond to the survey. All delegates were informed of the intention to collate responses as part of a research paper, and all responders consented for their responses to be included in this analysis. Responses were aggregated to provide summary statistics for the cohort of attendees.

\section{Results}

One hundred delegates attended the course and 89 provided complete responses. Fifty-four responders (60.7\%) worked in district hospitals and health centres across 10 different districts in the Southern Region of Malawi, and 35 (39.3\%) were from QECH and $\mathrm{KCH}$ (Table 1). Thirty-six (40.4\%) were nurses, $25(28.1 \%)$ were clinical officers or medical assistants, and 24 (30\%) were physicians, with four delegates $(4.4 \%)$ not documenting their occupations. Twenty-two percent of all healthcare workers had never received teaching on any aspect of renal disease. This was higher in district workers (28\% overall, $0 \%$ physicians, 30\% clinical officers and medical assistants, 33\% nurses) and nurses (31\%). If teaching occurred, it usually occurred at the undergraduate level $(76 \%)$, and more than three years previously $(66 \%)$. Fifty percent of healthcare workers overall had never received teaching on AKI; this was higher in district hospital staff $(63 \%$ overall, $10 \%$ physicians, $78 \%$ clinical officers and medical assistants, $78 \%$ nurses) and nurses (61\%) (Figure 1). If teaching occurred, again, it was usually at the undergraduate level (65\%). Despite this, 69\% of healthcare workers had managed patients with AKI, with 27\% seeing such patients on at least a weekly basis. Prior to the course, $44 \%$ of workers did not feel confident managing AKI; this was the case in $52 \%$ of district workers. Ninety-eight percent of all responders (including 100\% of nurses) wanted more support in managing patients with kidney disease.

There was limited knowledge of the renal service available at QECH, particularly among district hospital staff. Prior to the course, $68 \%$ of district workers were aware of the renal service at QECH, 19\% were aware of the adult renal clinic, and $9 \%$ were aware of the paediatric renal clinic (Figure 2). Thirty-nine percent and $14 \%$ of all healthcare workers had referred patients to the adult and paediatric clinics, respectively. Thirty-four percent of healthcare workers were unaware that haemodialysis is available for AKI at QECH (including 55\% of district hospital staff), and 53\% were unaware that peritoneal dialysis is available for AKI (including 74\% of district hospital staff). Only 33\% had referred a patient with AKI to QECH.

\section{Discussion}

AKI is common in Malawi, and management across different healthcare settings is suboptimal. Consequently, AKI has negative outcomes for both individuals and society. This study describes healthcare workers' experience and education related to AKI within Malawi. The healthcare workers
Table 1: Details of healthcare workers who responded to survey

\begin{tabular}{lccccc}
\hline District and non-QECH Blantyre attendees & & & \\
\hline District & Nurses & $\begin{array}{l}\text { Clinical officers } \\
\text { and medical } \\
\text { assistants }\end{array}$ & Clinicians & $\begin{array}{l}\text { Not } \\
\text { documented }\end{array}$ & Total \\
\hline Balaka & 2 & 1 & 0 & 1 & 4 \\
Blantyre & 1 & 2 & 1 & 1 & 5 \\
Chikwawa & 0 & 2 & 1 & 0 & 3 \\
Machinga & 4 & 3 & 0 & 0 & 7 \\
Mangochi & 3 & 2 & 2 & 0 & 7 \\
Mulanje & 3 & 0 & 2 & 0 & 5 \\
Neno & 3 & 3 & 1 & 0 & 7 \\
Nkhata Bay & 0 & 0 & 1 & 0 & 1 \\
Thyolo & 1 & 3 & 2 & 1 & 7 \\
Zomba & 2 & 6 & 0 & 0 & 8 \\
\hline Districts total & 19 & 22 & 10 & 3 & 54 \\
\hline
\end{tabular}

\begin{tabular}{lcccccc|}
\hline QECH and KCH attendees & & & & \\
\hline QECH/KCH total & 17 & 3 & 14 & 1 & 35 \\
\hline Overall total & $\mathbf{3 6}$ & $\mathbf{2 5}$ & $\mathbf{2 4}$ & $\mathbf{4}$ & $\mathbf{8 9}$ \\
\hline $\mathrm{QECH}=$ Queen Elizabeth Central Hospital, Blantyre & &
\end{tabular}

$\mathrm{QECH}=$ Queen Elizabeth Central Hospital, Blantyre

$\mathrm{KCH}=$ Kamuzu Central Hospital, Lilongwe

Figure 1: Percentages of nurses, district hospital workers, and all healthcare workers who had no previous teaching on $A K I$

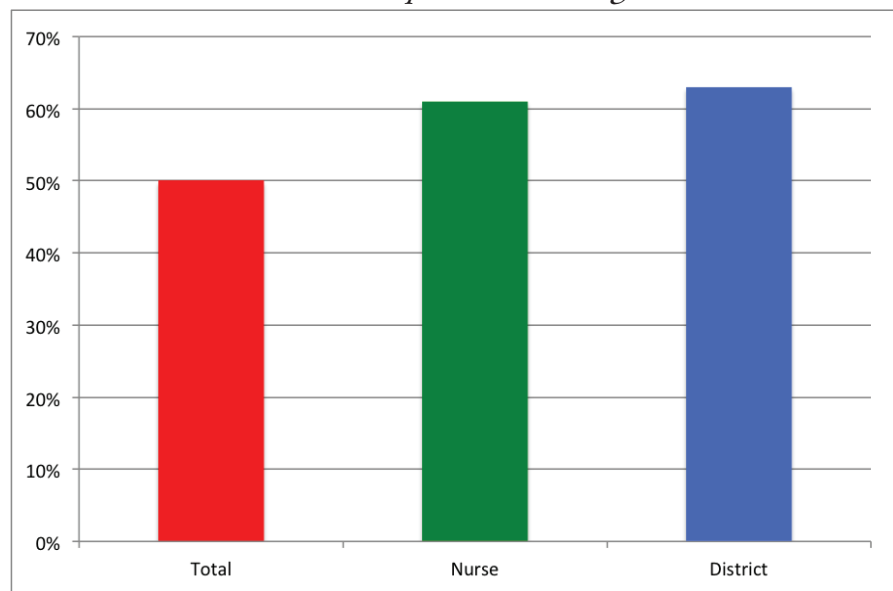

Figure 2: Percentages of district healthcare workers who were aware of the renal service at QECH, the adult nephrology clinic at QECH, and the paediatric nephrology clinic at QECH

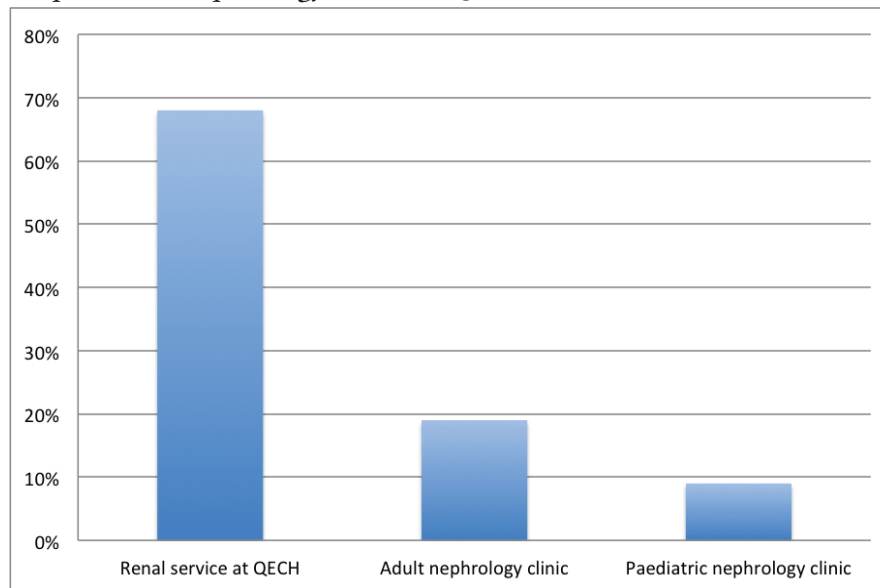

surveyed were from a variety of different backgrounds, both in terms of their roles and where they work. Healthcare workers from all settings are encountering patients with AKI frequently within Malawi but have received limited education and training. This is particularly the case among district clinical officers and nurses, who lack specific teaching on AKI in their respective curricula. What little they have received has almost exclusively occurred during undergraduate training and, even then, exposure to AKI is limited. This may, in part, be because of limited access to laboratory estimates of renal function (creatinine and urea) across many settings within Malawi. Only $56 \%$ of responders felt confident in managing $\mathrm{AKI}$ and almost all healthcare workers wanted more support http://dx.doi.org/10.4314/mmj.v27i3.6 
in managing patients with kidney disease. Limited knowledge and education in renal disease is not restricted to our setting. A recent survey of junior doctors within the UK demonstrated that $50 \%$ of trainees could not define AKI, 30\% could not name more than two risk factors for AKI, and $37 \%$ could not name appropriate indications for renal referral. ${ }^{7}$ Scores were lowest in the most junior trainees. Similarly, in the US, internal medicine residents have been shown to lack awareness of the need for timely referral to nephrology services, or basic facts on $\mathrm{CKD}^{8,}{ }^{8}$ Despite the need for more support, there was limited knowledge of the renal services available at $\mathrm{QECH}$, specifically the adult and paediatric renal clinics, and the dialysis services that are available for the treatment of AKI. This was particularly the case among district healthcare workers and despite promotional efforts of the renal services team at QECH. High staff turnover may explain some of the lack of awareness, and this poses challenges for establishing a sustainable knowledge base for the management of AKI in Malawi. We aim to continue to promote the renal services at QECH to ensure we provide as much support as we can to renal patients across southern Malawi. Our objective is to facilitate an improved referral system, and provide feedback to referring teams as part of a combined education process. But do more intensive educational efforts improve healthcare workers' confidence and ability in managing AKI? More importantly, does this lead to improvements in patient care? At the end of our symposium, feedback demonstrated that delegates did feel more confident in managing AKI and we plan to conduct further studies to establish whether enhanced training in AKI translates to better patient care in Malawi. Elsewhere, education has been shown to improve outcomes with an intensive AKI education programme in Leicester, UK demonstrating improved clinical practice in two large teaching hospitals. ${ }^{10}$ We plan to expand our educational efforts to include healthcare workers of all levels from all healthcare settings in Malawi. We aim to deliver the AKI symposium yearly, to run smaller AKI education sessions within districts across Malawi, to introduce AKI teaching to all students and junior doctors at department inductions, and to develop an online AKI learning module specifically adapted to the management of AKI in a sub-Saharan African setting. To determine the efficacy of this teaching, we aim to assess patient outcomes before and after these teaching interventions. We are in the process of opening a dedicated AKI unit at QECH, which will act not only as a centre of clinical excellence in the specialist management of AKI, but also as a training hub for educating healthcare workers of all levels in the detection and management of AKI. In conclusion, this study highlights deficiencies in education about, and clinical experience in, the management of AKI among Malawian healthcare workers. It is imperative we act to improve support for all healthcare workers caring for patients with this preventable and curable condition within our healthcare system.

\section{References}

1. Lewington AJP, Cerdá J, Mehta RL. Raising awareness of acute kidney injury: a global perspective of a silent killer. Kidney Int. 2013 Sep;84(3):457-67.

2. Wonnacott A, Meran S, Amphlett B, Talabani B, Phillips A. Epidemiology and outcomes in community-acquired versus hospitalacquired AKI. Clin J Am Soc Nephrol CJASN. 2014 Jun 6;9(6):100714.

3. Nash K, Hafeez A, Hou S. Hospital-acquired renal insufficiency. Am J Kidney Dis Off J Natl Kidney Found. 2002 May;39(5):930-6.

4. Susantitaphong P, Cruz DN, Cerda J, Abulfaraj M, Alqahtani F, Koulouridis I, et al. World Incidence of AKI: A Meta-Analysis. Clin J Am Soc Nephrol. 2013 Jun 9;8(9):1482-93.

5. Chertow GM, Burdick E, Honour M, Bonventre JV, Bates DW. Acute kidney injury, mortality, length of stay, and costs in hospitalized patients. J Am Soc Nephrol JASN. 2005 Nov;16(11):3365-70.

6. Evans, R. [Four-week audit on the assessment and management of renal impairment in patients admitted to the general medical wards at Queen Elizabeth Central Hospital, Blantyre, Malawi, 2015 Feb-Mar]. Unpublished raw data. College of Medicine, University of Malawi, Blantyre, Malawi.

7. Muniraju TM, Lillicrap MH, Horrocks JL, Fisher JM, Clark RMW, Kanagasundaram NS. Diagnosis and management of acute kidney injury: deficiencies in the knowledge base of non-specialist, trainee medical staff. Clin Med Lond Engl. 2012 Jun;12(3):216-21.

8. Agrawal V, Ghosh AK, Barnes MA, McCullough PA. Perception of indications for nephrology referral among internal medicine residents: a national online survey. Clin J Am Soc Nephrol CJASN. 2009 Feb;4(2):323-8.

9. Agrawal V, Ghosh AK, Barnes MA, McCullough PA. Awareness and knowledge of clinical practice guidelines for CKD among internal medicine residents: a national online survey. Am J Kidney Dis Off J Natl Kidney Found. 2008 Dec;52(6): 1061-9.

10. Xu G, Baines R, Westacott R, Selby N, Carr S. An educational approach to improve outcomes in acute kidney injury (AKI): report of a quality improvement project. BMJ Open. 2014;4(3):e004388. 